\title{
Paediatric lung imaging: the times they are a-changin'
}

\author{
Harm A.W.M Tiddens ${ }^{1,2}$, Wieying Kuo ${ }^{1,2}$, Marcel van Straten ${ }^{2}$ and \\ Pierluigi Ciet ${ }^{1,2}$
}

Affiliations: ${ }^{1}$ Pediatric Pulmonology and Allergology, Erasmus MC - Sophia Children's Hospital, University Medical Centre, Rotterdam, The Netherlands. ${ }^{2}$ Radiology and Nuclear Medicine, Erasmus University Medical Center - Sophia Children's Hospital, Rotterdam, The Netherlands.

Correspondence: H.A.W.M Tiddens, Pediatric Pulmonology and Allergology, Erasmus MC - Sophia Children's Hospital, University Medical Center, Rotterdam, 3015 CE, The Netherlands. E-mail: h.tiddens@erasmusmc.nl

@ERSpublications

CT and MRI are increasingly replacing lung function tests to diagnose and monitor lung diseases in young children http://ow.ly/6KSb30hRdEz

Cite this article as: Tiddens HAWM, Kuo W, van Straten M, et al. Paediatric lung imaging: the times they are a-changin’. Eur Respir Rev 2018; 27: 170097 [https://doi.org/10.1183/16000617.0097-2017].

ABSTRACT Until recently, functional tests were the most important tools for the diagnosis and monitoring of lung diseases in the paediatric population. Chest imaging has gained considerable importance for paediatric pulmonology as a diagnostic and monitoring tool to evaluate lung structure over the past decade. Since January 2016, a large number of papers have been published on innovations in chest computed tomography (CT) and/or magnetic resonance imaging (MRI) technology, acquisition techniques, image analysis strategies and their application in different disease areas. Together, these papers underline the importance and potential of chest imaging and image analysis for today's paediatric pulmonology practice. The focus of this review is chest CT and MRI, as these are, and will be, the modalities that will be increasingly used by most practices. Special attention is given to standardisation of image acquisition, image analysis and novel applications in chest MRI. The publications discussed underline the need for the paediatric pulmonology community to implement and integrate state-of-the-art imaging and image analysis modalities into their structure-function laboratory for the benefit of their patients.

\section{Introduction}

Until recently, functional tests were the most important tools for the diagnosis and monitoring of lung diseases in the paediatric population. Functional tests are an indirect method to detect structural lung changes and are relatively insensitive for the detection and monitoring of localised structural lung changes. Chest imaging has gained importance in paediatric pulmonology as a diagnostic and monitoring tool to evaluate lung structure, thanks to technical innovations in computed tomography (CT) and magnetic resonance imaging (MRI) technology. When searching PubMed using the keywords "lung", "child" and "imaging", over 300 papers have been published since January 2016. Around 220 of these papers include chest CT or MRI acquisition techniques, image analysis strategies and their application in different disease areas. Of these papers $80 \%$ were related to chest CT and $20 \%$ to chest MRI. Together, these papers underline the importance and potential of chest imaging and image analysis in today's paediatric pulmonology practice. The focus of this review is on chest CT and MRI, as these are the modalities where considerable progress has been made over the past year. For the role of chest ultrasound, positron emission tomography (PET)-CT and PET-MRI, we refer to recent comprehensive reviews [1-4]. Together,

Received: Aug 242017 | Accepted after revision: Dec 132017

Conflict of interest: Disclosures can be found alongside this article at err.ersjournals.com

Provenance: Commissioned article, peer reviewed.

Copyright CERS 2018. ERR articles are open access and distributed under the terms of the Creative Commons Attribution Non-Commercial Licence 4.0. 
these publications underline the need for the paediatric pulmonology community to re-evaluate the role of the various modalities for the benefit of their patients. The current gap between the world of imaging and functional tests needs to be bridged. We will briefly discuss the historical context of recent developments, followed by key developments in chest CT, MRI and image analysis in the past year.

\section{Of sound and vision}

Up to 1945, the stethoscope (invented by R. Laennec) was the most important diagnostic tool for investigating the lung, but then the chest radiograph was accepted by the medical community as a more sensitive diagnostic tool to evaluate lung structure. This paradigm shift, nearly 50 years after the discovery of X-rays by W. Röntgen, is nicely documented by a landmark paper published in 1945 by the Royal College of Medicine, which summarises the pros and cons of chest radiographs versus the stethoscope [5, 6]. Even though major limitations of chest radiographs were recognised at that time, chest radiography has been widely used ever since as a diagnostic tool to depict lung structure for the detection of lung disease. The next major breakthrough came when Cormack and Hounsfield developed CT. The first CT scanner for clinical use was installed in Cambridge in 1971. The first papers describing the use of chest CT in the paediatric age group began to appear a few years later, in 1977 [7]. Despite its enormous potential in depicting lung structure, its adaption as a diagnostic tool for paediatric pulmonology has been slow. An important argument against the use of CT in the paediatric population related to its relatively high radiation burden compared to CXR. Fortunately, over recent decades, technical innovations in CT scanner technology and image reconstruction have resulted in a substantial reduction in the radiation dose [8]. Powerful post-processing techniques, such as iterative image reconstruction, allowed reduction of the radiation dose to levels which are nowadays in the order of 3-6 months background radiation [9]. Furthermore, the possible risks related to radiation, as well as the perception of these risks, are now clearly described and put into perspective, allowing risk and benefit to be balanced more adequately, without pitfalls [10-14]. Furthermore, thanks to the development of very fast CT scanners, it is now possible to scan even rapidly breathing young children without the need for anaesthesia or sedation. As a result of these innovations, chest CT can now be used more safely in the paediatric population to diagnose and monitor a wide range of lung diseases. However, the more widespread use of chest CT requires standardisation of chest CT protocols and breathing manoeuvres to a similar level as has been accomplished for lung function tests. The paediatric pulmonology community has to take on this responsibility, together with the radiology community, to accomplish lung volume standardisation.

\section{Every breath you take}

Standardisation of breathing manoeuvres for lung function tests has been well implemented throughout the world. This contrasts sharply with the lack of standardisation for the acquisition of chest CT images. It has long been recognised that spirometry guidance to standardise lung volume during chest CT can be important for the proper diagnosis of bronchiectasis and parenchymal diseases on inspiratory images, as well as for the recognition of malacia and regions of low attenuation on expiratory scans $[15,16]$. Spirometry-guided CT improves the diagnostic yield, in particular that of the expiratory chest CT. It has even been suggested that for cystic fibrosis (CF), an expiratory scan might suffice for the diagnosis of all relevant pathological changes [17]. Despite these studies showing the potential benefit of spirometry-guided image acquisition, it has not been implemented on a wide scale to date. New studies have been published that improve our understanding of the potential benefit of spirometry-guided chest imaging. To implement such image acquisition in the clinic, close collaboration between pulmonologists and radiologists is required. Its feasibility for the clinical routine has been described by SALAMON et al. [18], who outline a practical method to obtain accurate lung volume measurements for the guidance of chest CT imaging in paediatric patients. This procedure requires training of the subject before each chest CT or MRI, an MRI-compatible spirometer, and close collaboration between a lung function technician and the radiographer. Patients are trained to execute a breath-hold with open glottis at total lung capacity level for the inspiratory scan and at residual volume for the expiratory scan. A good-to-excellent target volume level for the inspiratory or expiratory scan was achieved in $\sim 90 \%$ of children. Spirometry-guided chest CT scans have been evaluated in clinical practice in a tertiary care children's hospital [19]. In this retrospective casecontrol study in children aged $\geqslant 8$ years, CT scans obtained before and after implementation of a spirometry-guided CT protocol were compared. Spirometry-guided CT scans ( $\mathrm{n}=50$ cases) were matched by age, sex and diagnosis (CF versus other) to CT scans obtained with voluntary breath-holds in the 6 years before implementation of the spirometry assistance protocol (controls). CT scans were evaluated by two paediatric radiologists blinded to the study. The most important difference was in the mean $\pm \mathrm{SD}$ expiratory image density, which was $-629 \pm 95 \mathrm{HU}$ among cases and $-688 \pm 83 \mathrm{HU}$ among controls $(\mathrm{p}=0.002)$. The authors concluded that spirometry-assisted CT scans had a significantly greater difference in lung density between inspiratory and expiratory scans than those performed with voluntary 
breath-holds, thus probably improving the ability to detect air trapping. No appreciable difference in image quality was detected for the presence of motion artefacts or atelectasis.

It has been established that it is feasible for the spirometry-assisted method to be used successfully in patients suffering from Pompe disease to determine the function of the diaphragm [20]. Furthermore, spirometry-assisted MRI has even been used in a large birth cohort study. In this study, two spirometry-controlled inspiratory and two expiratory MRI scans were acquired within 5 min with a success rate of $90 \%$ [21]. Hence, implementation of spirometry-guided chest CT and MRI is considered feasible and adds to the diagnostic quality of images. However, it requires the involvement of a lung function technician, and there are some logistic hurdles to overcome. The need for standardisation is not dissimilar from that of lung function measurements, where proper training adds to the reproducibility and interpretation of the test. This developments needs to be driven by the paediatric pulmonology community, as lung function technicians play a key role in the implementation and execution of procedures.

\section{Let's stick together}

To date, the implementation of CT protocols has been largely performed by local radiology communities and has been focused on the balance between diagnostic image quality and radiation dose levels [22]. There is a great need for more standardisation of CT protocols between centres, for a number of reasons. First, for rare lung diseases such as CF, primary ciliary dyskinesia (PCD), bronchiectasis and interstitial lung diseases, large global clinical networks and registries have been developed to improve our understanding and treatment of these diseases. In these rare disease communities there is a desire to add information obtained from images to registries. This can be accomplished best when imaging protocols are sufficiently standardised to allow centralised scoring, and manual or automated image analysis. The latter requires a higher level of standardisation. Secondly, chest CT is increasingly used as an outcome measure in clinical trials for various chest diseases. Clinical trial networks have been set up for the joint execution of such clinical trials. So far, standardising image quality across centres in relation to radiation dose, reconstruction kernels, slice thickness, etc. has not been well addressed. Recognising the need for a higher level of standardisation of chest CT, the Standardised Chest Imaging Framework for Interventions and Personalised Medicine in CF (SCIFI CF) was founded to characterise chest CT image quality and radiation doses among 16 CF centres in the European Union (EU), seven in Australia and three in the USA [22]. The authors aimed to standardise CT protocols in children and adolescents in several CF centres. In doing so, an image quality (Q-factor) is assessed; this incorporates the influence of both dose and spatial resolution on image quality. Across the $16 \mathrm{EU}$ centres CT protocols varied greatly. However, when adjusting for differences in preferred spatial resolution and radiation dose, the performance of all CT scanners (i.e. the Q-factor) was found to fall within a small range. It was concluded that multicentre standardisation of chest CT in children and adolescents with CF is achievable for clinical care and management. The SCIFI effort has contributed to the inclusion of chest CT as a primary or secondary outcome measure in several ongoing clinical trials in CF (e.g. clinicaltrials.gov NCT02950883 and NCT01270074). Another important standardisation effort has been the completion of guidelines to homogenise radiation dose for paediatric imaging throughout Europe [23]. Both efforts on image quality and radiation dose standardisation were carried out in concordance with the protocol optimisation principle ALARA (As Low As Reasonably Achievable).

To standardise chest MRI across centres and vendors to the level that it can be used for registries and multicentre clinical trials is considered a major challenge, and it is still early days. There are a number of reasons for this. First, there are endless possibilities to vary the settings for the sequences. Secondly, there are substantial differences in the sequences that can be used routinely between vendors. Chest MRI is currently mostly used in single-centre studies. Methods to standardise image quality for MRI are in development and are being applied to allow multicentre studies (clinical trial.gov NCT02270476 and NCT01245933).

\section{When numbers get serious}

Today's radiology reports of chest images for routine care are still largely expert-based, mostly not standardised, and do not contain quantitative outcome data. In other clinical specialities like cardiology, quantitative post-processing methods to acquire objective outcome measures are available and well implemented. For lung diseases in adults, such as chronic obstructive pulmonary disease (COPD), image analysis systems have been developed to measure airway dimensions, parenchymal density and emphysema $[24,25]$. Scoring systems have also been used to characterise disease progression in a cohort of PCD patients [26]. Initially, these systems were developed for research purposes, but increasingly they are finding their way into routine clinical care. 
In children, scoring systems have been extensively used to validate chest CT outcome measures in CF [27]. Using the standardised CF-CT scoring system it was shown that the sweat test is an early predictor of later structural lung disease [28]. Unfortunately, scoring systems such as the CF-CT system are not very sensitive for the detection and monitoring of early disease and cannot be automated easily. PRAGMA-CF (Perth-Rotterdam Annotated Grid Morphometric Analysis for CF) is a quantitative grid method to quantify morphological changes that occur in early CF disease [29], and is based on a morphometric approach previously used for quantification of advanced CF lung disease. Comparing lung clearance index (LCI) values to PRAGMA-CF outcomes in 42 infants, 39 preschool and 38 school-aged children, it was concluded that for infants LCI is insensitive for the detection of structural lung disease and that in preschool and school-age children LCI cannot replace chest CT to screen for bronchiectasis [30].

A morphometric approach similar to that of PRAGMA-CF is now also applied to other diseases in children and adults, such as bronchopulmonary dysplasia (BPD) [31, 32] and interstitial lung diseases [33].

Airway disease is an important component of the above-mentioned diseases. For this reason Kuo and co-workers $[34,35]$ developed the airway-artery (AA) method to measure all visible airway-artery pairs in a view perpendicular to the airway axis on a 3D-reconstructed bronchial and arterial tree. The AA method allows an objective diagnosis of bronchiectasis and airway wall thickening. KUO and co-workers [34, 35] compared airway and artery dimensions in a small group of preschool [35] and school-aged [34] children with CF and in controls (figure 1). Depending on the age of the patients and the inspiratory level during CT acquisition, between 50 and 500 AA pairs could be measured per CT. In school-aged children the number of visible AA pairs was doubled in patients with CF compared to controls due to inflammation and dilation of the smaller airways augmenting their visibility. The diagnosis of bronchiectasis appeared to

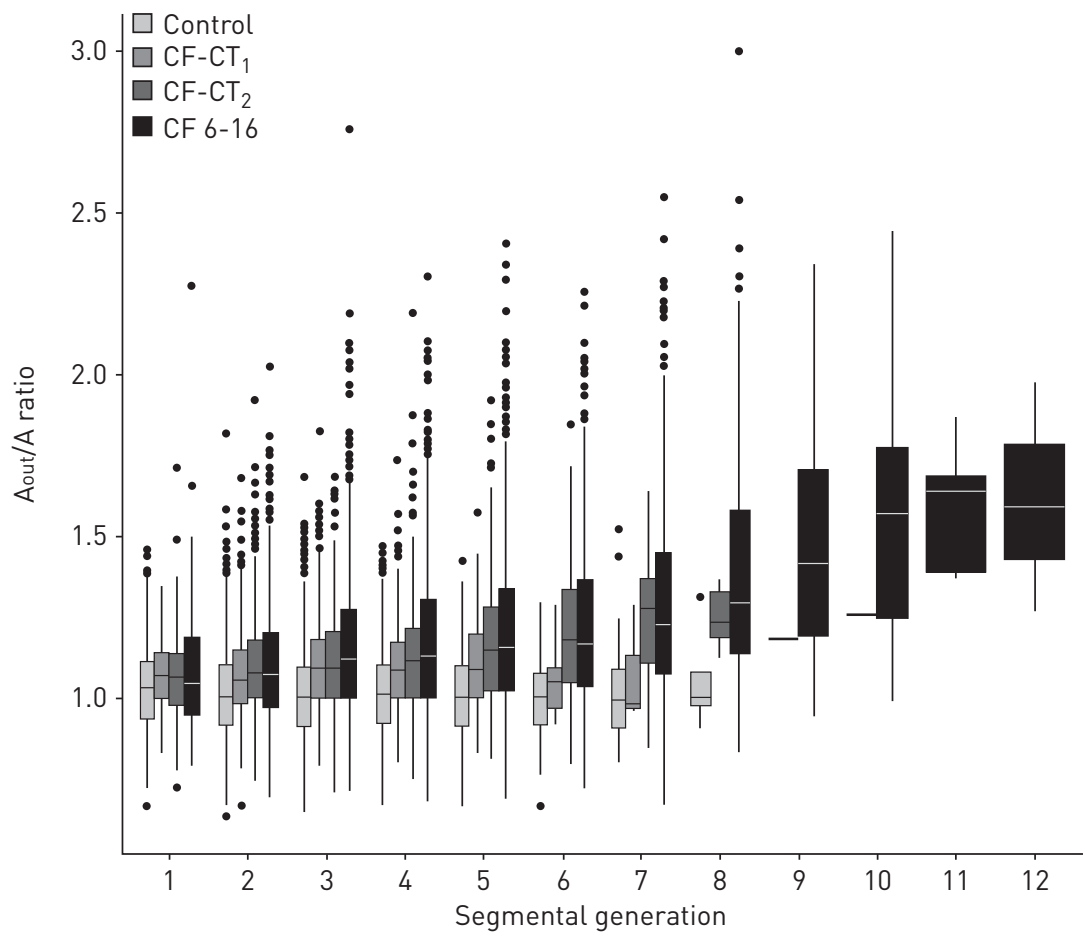

FIGURE 1 A method for the objective assessment of airway artery dimensions (the AA method) to diagnose bronchiectasis. Boxplots show the ratio between the outer edge of the airway (Aout) and the adjacent artery (A). Aout/A ratios are shown for four consecutive groups: control ( $n=23$ ); first and second cystic fibrosis-computed tomography (CF-CT $(n=12)$ and $C F-C T_{2}(n=12)$, respectively); and $C T$ scans in a cross sectional cohort including patients aged $6-16$ years (CF 6-16) (n=11). In total, 11262 AA pairs were measured. Aout/A ratios are plotted against segmental generation (1 is the first segmental bronchus up to the 12th airway generation peripheral from the segmental bronchus). Control subjects were age-matched for CF patients. Median ages are 2 years, 3.9 years and 11 years for $C F-C_{1}, C F-C_{2}$ and $C F$ 6-16, respectively. Boxes show median (horizontal line), interquartile range (box) and 1.5x interquartile range (whiskers). Outliers are shown as points. Note that for controls, Aout/A ratios were constant, whereas for each of the three CF groups an increasing and significant difference could be found in Aout/A ratio between the CF and control group from generation 2 to generation 5 (all $p \leqslant 0.02$ ). Furthermore, the difference between CF and controls was bigger for the oldest CF patients. Note that for generation 9 and higher, no more airway artery pairs were visible on the scans for control subjects, while airway artery pairs were still visible on the CT scans of CF patients [34, 35]. Reproduced and modified from [34]. 
be dependent on the lung volume at which the CT scan was acquired [35]. These findings once more support the need for volume standardisation for cooperative children during acquisition. In addition, it objectively showed that a comparison of the outer airway diameter with the artery is more accurate in assessing bronchiectasis than the inner airway diameter. Finally, Kuo et al. [36] showed a good correlation between the AA method and the PRAGMA-CF and CF-CT scoring methods. Unfortunately, manual execution of the AA method is very time consuming. For this reason algorithms are in development that will allow sensitive analysis of airway dimensions for the diagnosis of airway wall thickening and bronchiectasis [37]. Adding quantitative information on lung CT scans to the routine radiology report is close at hand, and will be an important step forward for the diagnosis and monitoring of lung diseases. How quantitative CT markers can be used as outcomes in clinical CF studies has been described in a review and in comments as part of a special series in the Journal of Cystic Fibrosis [38-40]. Combining imaging and functional outcomes in clinical studies will be important to improve our understanding of the effectiveness of novel therapies, such as the very costly cystic fibrosis transmembrane conductance regulator (CFTR) correctors and potentiators.

\section{Radio Ga Ga}

MRI is sometimes described as making pictures with a radio. Will MRI replace CT at some point as the leading technique for making images of the lung? The first MRI prototype, a radiation-free alternative to chest CT, was installed in 1977. Although MRI has revolutionised medicine in many disease areas, its use for lung diseases has lagged behind. The reasons for this include the low-proton density of lung tissue, the continuous motion of the lung, and the elevated air content, which results in low signal intensity and fast signal decay. In 1983 the first study was published that included chest MRIs in children [41]. Major innovations in chest MRI in paediatrics have taken place over the past decade, especially in relation to the hardware. Fast acquisition techniques, respiratory gating and new high-resolution techniques continue to close the image-quality gap between CT and MRI [42].The resolution of conventional ${ }^{1} \mathrm{H}-\mathrm{MRI}$ for morphologic imaging is relatively poor, and is inferior to that of chest CT [43]. However, image resolution has improved considerably over the past decade thanks to the development of novel ultrashort echo (UTE) (figure 2) and zero echo time (ZTE) sequences, which allow submillimetre high-resolution images to be obtained [44, 45]. These UTE/ZTE sequences have been applied in quiet breathing neonates [46].

These sequences have been compared to CT in a group of infants with lung diseases, showing that the lung signal intensity of UTE correlates highly with lung density measured by CT [46]. This would allow the introduction of quantitative MRI parameters to define lung pathology, such as trapped air and emphysema, as is routinely done for asthma and COPD patients. Furthermore, to obtain functional information on lung perfusion an intravenous contrast agent can be applied [47], although the use of contrast agents for paediatric patients is still debated after evidence of gadolinium deposition in the body [48]. Alternative MRI techniques could be applied that allow simultaneous perfusion and ventilation imaging without using contrast [49]. Another interesting development to improve resolution is the use of inhaled hyperpolarised gases such as ${ }^{129} \mathrm{Xe}$ or ${ }^{3} \mathrm{He}$, and other inhaled contrast agents to enhance the spatial resolution of lung airspaces [50]. The heterogeneity of ventilation can be assessed and hypoventilated areas can be easily identified. Although these techniques add complexity to the procedure and will increase the challenge of standardisation, they can be of great value as a research tool to improve
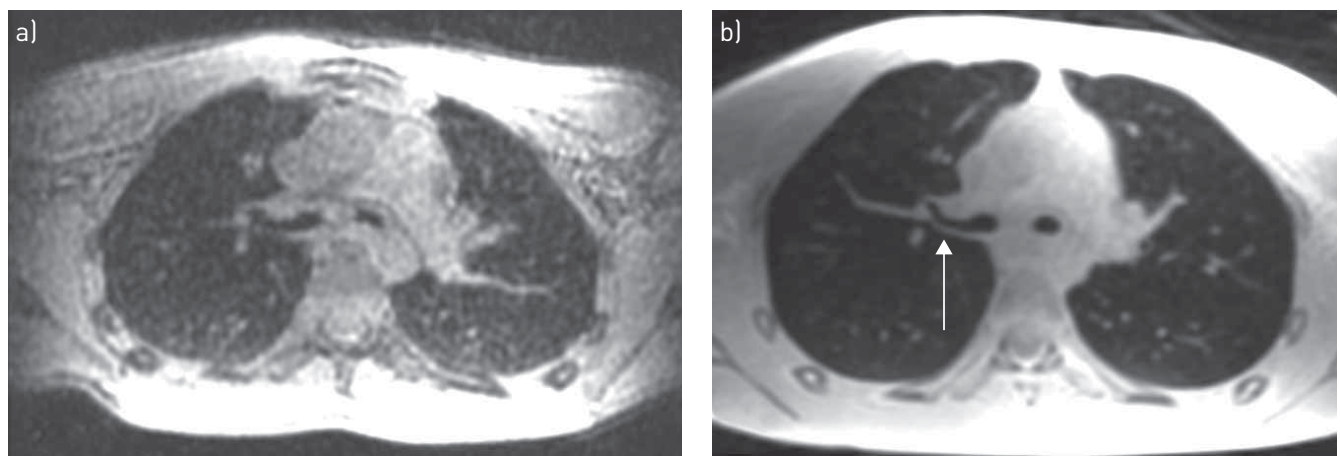

FIGURE 2 Chest magnetic resonance images of a 15-year-old boy with asthma. Comparison between 3D spoiled gradient echo (SPGR) and 3D ultra-short echo time (UTE) at 3 T (Dicovery MR750; GE Healthcare, Chicago, IL, USA). a) $2 \times 2 \times 2 \mathrm{~mm}$ end-expiratory breath-hold axial 3D SGPR (TE $=0.748 \mathrm{~ms}) ;$ b) $2 \times 2 \times 2 \mathrm{~mm}$ end-expiratory respiratory-triggered $3 \mathrm{D}$ UTE $(T E=0.032 \mathrm{~ms})$. Note the higher resolution and signal-to-noise ratio of UTE with better definition of the airways and air wall (arrow). 
our understanding of various lung diseases and to evaluate the efficacy of novel drugs. This technique has been applied in small cross-sectional studies in CF [51-55] and in asthma [56]. However, for clinical management in $\mathrm{CF}$ it might be sufficient to identify and quantify low-intensity regions related to hypoperfusion and/or trapped air using spirometer-controlled expiratory ${ }^{1} \mathrm{H}-\mathrm{MRI}$ [18] or other strategies such as normalised T1 and non-contrast perfusion techniques [57]. This needs to be further investigated in comparative studies.

Hyperpolarised ${ }^{3} \mathrm{He}$ can also be used to assess alveolar size. This has been applied in BPD [58]. In a small follow-up study in 16 BPD patients, alveolar size was higher than that of former healthy term-born patients.

Another important advantage of chest MRI over chest CT is that it allows us to acquire simultaneous information on lung structure and function without using ionising radiation. This opens up new ways to study lung mechanics in asthmatic subjects [59].

Another exciting novel application is the potential of MRI to visualise lung inflammation and infection. In a relatively large single-centre study, conventional MRI sequences were used to assess paediatric pulmonary infection [60]. MRI had comparable sensitivity and specificity to CT for the diagnosis and monitoring of lung infection. Interesting methods have been developed that could facilitate diagnosing allergic bronchopulmonary aspergillosis (ABPA) in CF [61]. Furthermore, MRI could be particularly important for paediatric patients who cannot be exposed to ionising radiation, or in immunocompromised children who have repeated infections [62]. For lung inflammation detection and monitoring, diffusion-weighted MRI has been used in CF patients with pulmonary exacerbation [63]. Many of the MRI innovations described above were initially developed for CF and are now also applied for research in asthma [64], immunocompromised children [62], pulmonary infections [65], tuberculosis [66], BPD [46, 58], congenital diaphragmatic hernia [67, 68], pulmonary sarcoidosis [69], and even in the follow-up of a large birth cohort study [21]. Chest MRI is increasingly used for clinical management in the follow-up of CF lung disease [43] or for the assessment of central airway dynamics and dimensions [70]. The major challenge for MRI to make it into the daily clinic is standardisation of MRI protocols across centres and vendors. This will require a comparison of protocols using phantoms and selection and validation of comparable vendor-specific sequences.

\section{Report, numbers? Ch-ch-ch-ch changes}

For conventional MRI, quantification of morphological changes is more challenging than in CT as its resolution is lower than that of CT. Using scoring techniques to evaluate ${ }^{1} \mathrm{H}-\mathrm{MRI}$ images of 57 patients it was shown, as in previous studies, that ${ }^{1} \mathrm{H}$-MRI underestimates mild CF disease and overestimates severe CF disease compared to CT $[43,70]$. In two small cross-sectional studies in paediatric and adult CF patients using UTE-MRI, chest MRI scores correlated well with chest CT scores [44, 61]. Scoring was also used to correlate MRI outcomes to LCI in a cross-sectional study that included 97 stable children with CF aged 0.2-21 years. Overall, correlations were weak but significant. In addition, 25 children had an MRI before and after therapy that showed significant improvements in several MRI scores except for airway wall thickness scores [47]. ${ }^{1} \mathrm{H}$-MRI has also been used to characterise mucus using differences in its intensity between T1 and T2 weighted images as a method to identify patients at risk for developing ABPA [61].

These promising but discordant results warrant further longitudinal studies comparing the sensitivity for tracking structural CF lung disease using these improved MRI sequences with that for chest CT. In addition, it will be necessary to develop more sensitive semi-automated image analysis techniques to replace the currently used coarse scoring techniques. Only with a sufficiently standardised multi-vendor, multicentre and multi-sequence MRI protocol will we be able to promote the use of chest MRI on a larger scale.

For quantification of images using inhaled hyperpolarised noble gases as a contrast agent, ventilation defects are counted, or the volume of ventilation defects is computed and expressed as a fraction of total lung volume. Using hyperpolarised ${ }^{3} \mathrm{He}-\mathrm{MRI}$, Altes et al. [55] showed (in a small pilot study) a reduction in the fraction of poorly ventilated lung tissue in CF patients while on treatment with the CFTR potentiator ivacaftor. On stopping treatment after 48 weeks, the volume of poorly ventilated lung tissue increased again to baseline values, showing a pattern similar to baseline MRI. This study suggests that ivacaftor can improve the ventilation of even structurally abnormal regions of the lung, but that these effects disappear after therapy is stopped. In using hyperpolarised noble gases to estimate alveolar size, the apparent diffusion coefficient is used as the outcome measure. In BPD patients, apparent diffusion coefficient values were significantly greater than in age-matched healthy controls, suggesting that, in the former, alveoli are enlarged [58].

Other quantitative functional parameters that can be extracted from MRI are central airway dimensions for the objective diagnosis of malacia [70-72]. Central airway diameters are measured at end-inspiration, 
during a forced expiration and at end-expiration to compute the change in cross-sectional area. Advantages of this MRI method include that it does not require general anaesthesia, unlike bronchoscopy, that the impact of a forced expiration and cough on airway diameter can be evaluated, and that it can be standardised well.

Another great application of chest MRI is that it can be used for quantitative analysis of the function of the diaphragm (being the most important respiratory muscle) [20]. Image registration and lung surface extraction are used to quantify lung kinematics during breathing. This allows us to compute the independent contributions of the diaphragm and thoracic muscles to the respiratory cycle. This quantitative method was used in a pilot study in Pompe patients and control subjects, and showed minimal motion of the diaphragm in the presence of mostly thoracic musculature movement in Pompe patients.

Using diffusion-weighted imaging, inflammation hotspots are visible either because free water movement is restricted due to the increased cellularity or due to an increase in microperfusion in relation to inflammation. The hotspots were counted over the course of an intravenous antibiotic treatment in CF patients treated for a pulmonary exacerbation, then compared to a control group of stable CF patients [63]. A striking finding was that at the end of treatment, hotspots were still visible in some patients. Moreover, quantitative diffusion-weighted imaging-derived parameters, such as the apparent diffusion coefficient, showed good sensitivity and specificity to detect respiratory tract exacerbations in CF patients. The ability of diffusion-weighted imaging-MRI to track inflammatory changes has great potential in assessing the efficacy of currently used exacerbation treatments and to develop more effective novel therapies.

There are many innovative MRI techniques to obtain detailed information on lung ventilation and perfusion, and a wealth of outcome measures can be extracted from these techniques. However, the greatest challenge is to select the most robust outcome measures and to validate these outcome measures. Importantly, such outcome measures should add novel information that impacts clinical decision making. Furthermore, the feasibility of implementing these techniques across centres using multiple MRI vendors needs to be established.

\section{Livin' in the future}

We have come a long way in our diagnostic capabilities since the invention of the stethoscope by Laennec. Innovations in lung imaging and image analysis will change the face of our diagnostic tool kit as we know it today. We are likely to continue to use the stethoscope, chest radiographs and lung function in daily practice, but their role and importance for patient care will once more change substantially in the coming decade thanks to the capabilities of today's state-of-the-art chest CT and the rapidly developing and exciting new capabilities of chest MRI. Novel chest CT, MRI and image analysis techniques will improve our understanding of the pathophysiology and treatment of lung diseases in the paediatric population. Although, initially, many of the developments were primarily focused on CF, they are now being applied to other diseases such as BPD, bronchiectasis, interstitial lung diseases, pneumonia, PCD, sarcoidosis, tuberculosis and congenital lung abnormalities. Close and structured collaboration between the pulmonology and radiology communities is needed to facilitate further standardisation efforts and for the development of reference values and automated image analysis of key outcome measures and for the validation of outcome measures.

\section{References}

1 Cox M, Soudack M, Podberesky DJ, et al. Pediatric chest ultrasound: a practical approach. Pediatr Radiol 2017; 47: 1058-1068.

2 Parisi MT, Bermo MS, Alessio AM, et al. Optimization of pediatric PET/CT. Semin Nucl Med 2017; 47: 258-274.

3 Capitanio S, Nordin AJ, Noraini AR, et al. PET/CT in nononcological lung diseases: current applications and future perspectives. Eur Respir Rev 2016; 25: 247-258.

Gatidis S, Bender B, Reimold M, et al. PET/MRI in children. Eur J Radiol 2017; 94: A64-A70.

Cheng TO. How Laennec invented the stethoscope. Int J Cardiol 2007; 118: 281-285.

Bennett TI. Discussion on the stethoscope versus X-rays. Proc R Soc Med 1945; 355: 7-9.

Vinocur CD, Dinn WM, Dudgeon DL. Computed tomographic scanning in children. J Pediatr Surg 1977; 12: $847-856$.

8 Lee C, Pearce MS, Salotti JA, et al. Reduction in radiation doses from paediatric CT scans in Great Britain. Br J Radiol 2016; 89: 20150305.

9 Greffier J, Pereira F, Macri F, et al. CT dose reduction using automatic exposure control and iterative reconstruction: a chest paediatric phantoms study. Phys Med 2016; 32: 582-589.

10 Kuo W, Ciet P, Tiddens HA, et al. Monitoring cystic fibrosis lung disease by computed tomography. Radiation risk in perspective. Am J Respir Crit Care Med 2014; 189: 1328-1336.

11 Brody AS, Guillerman RP. Don't let radiation scare trump patient care: 10 ways you can harm your patients by fear of radiation-induced cancer from diagnostic imaging. Thorax 2014; 69: 782-784.

12 Eisenberg JD, Lewin SO, Pandharipande PV. The fisherman's cards: how to address past and future radiation exposures in clinical decision making. Am J Roentgenol 2014; 202: 362-367. 
13 Tucker EW, Jain SK, Mahesh M. Balancing the risks of radiation and anesthesia in pediatric patients. $J$ Am Coll Radiol 2017; 14: 1459-1461.

14 Andronikou S. Letting go of what we believe about radiation and the risk of cancer in children. Pediatr Radiol 2017; 47: 113-115.

15 Robinson TE, Leung AN, Moss RB, et al. Standardized high-resolution CT of the lung using a spirometer-triggered electron beam CT scanner. Am J Roentgenol 1999; 172: 1636-1638.

16 Mott LS, Graniel KG, Park J, et al. Assessment of early bronchiectasis in young children with cystic fibrosis is dependent on lung volume. Chest 2013; 144: 1193-1198.

17 Loeve M, Lequin MH, de Bruijne M, et al. Cystic fibrosis: are volumetric ultra-low-dose expiratory CT scans sufficient for monitoring related lung disease? Radiology 2009; 253: 223-229.

18 Salamon E, Lever S, Kuo W, et al. Spirometer guided chest imaging in children: it is worth the effort! Pediatr Pulmonol 2017; 52: 48-56.

19 Otjen JP, Swanson JO, Oron A, et al. Spirometry-assisted high resolution chest computed tomography in children: is it worth the effort? Curr Probl Diagn Radiol 2018; 47: 14-18.

20 Mogalle K, Perez-Rovira A, Ciet P, et al. Quantification of diaphragm mechanics in Pompe disease using dynamic 3D MRI. PLoS One 2016; 11: e0158912.

21 Kooijman MN, Kruithof CJ, van Duijn CM, et al. The Generation R Study: design and cohort update 2017. Eur J Epidemiol 2016; 31: 1243-1264.

22 Kuo W, Kemner-van de Corput MP, Perez-Rovira A, et al. Multicentre chest computed tomography standardisation in children and adolescents with cystic fibrosis: the way forward. Eur Respir J 2016; 47: 1706-1717.

23 European Diagnostic Reference Levels for Paediatric Imaging. European Guidelines on DRLs for Paediatric Imaging. www.eurosafeimaging.org/wp/wp-content/uploads/2014/02/European-Guidelines-on-DRLs-for-PaediatricImaging_Revised_18-July-2016_clean.pdf Date last updated: March 08 2016. Date last accessed: Aug 17, 2017.

24 Nambu A, Zach J, Schroeder J, et al. Quantitative computed tomography measurements to evaluate airway disease in chronic obstructive pulmonary disease: relationship to physiological measurements, clinical index and visual assessment of airway disease. Eur J Radiol 2016; 85: 2144-2151.

25 Subramanian DR, Gupta S, Burggraf D, et al. Emphysema- and airway-dominant COPD phenotypes defined by standardised quantitative computed tomography. Eur Respir J 2016; 48: 92-103.

26 Shah A, Shoemark A, MacNeill SJ, et al. A longitudinal study characterising a large adult primary ciliary dyskinesia population. Eur Respir J 2016; 48: 441-450.

27 Tiddens HA, Rosenow T. What did we learn from two decades of chest computed tomography in cystic fibrosis? Pediatr Radiol 2014; 44: 1490-1495.

28 Caudri D, Zitter D, Bronsveld I, et al. Is sweat chloride predictive of severity of cystic fibrosis lung disease assessed by chest computed tomography? Pediatr Pulmonol 2017; 52: 1153-11414.

29 Rosenow T, Oudraad MC, Murray CP, et al. PRAGMA-CF. A quantitative structural lung disease computed tomography outcome in young children with cystic fibrosis. Am J Respir Crit Care Med 2015; 191: $1158-1165$.

30 Ramsey KA, Rosenow T, Turkovic L, et al. Lung clearance index and structural lung disease on computed tomography in early cystic fibrosis. Am J Respir Crit Care Med 2016; 193: 60-67.

31 van Mastrigt E, Logie K, Ciet P, et al. Lung CT imaging in patients with bronchopulmonary dysplasia: a systematic review. Pediatr Pulmonol 2016; 51: 975-986.

32 van Mastrigt E, Kakar E, Ciet P, et al. Structural and functional ventilatory impairment in infants with severe bronchopulmonary dysplasia. Pediatr Pulmonol 2017; 52: 1029-1037.

33 Jacob J, Bartholmai BJ, Rajagopalan S, et al. Evaluation of computer-based computer tomography stratification against outcome models in connective tissue disease-related interstitial lung disease: a patient outcome study. BMC Med 2016; 14: 190

34 Kuo W, de Bruijne M, Petersen J, et al. Diagnosis of bronchiectasis and airway wall thickening in children with cystic fibrosis: objective airway-artery quantification. Eur Radiol 2017; 27: 4680-4689.

35 Kuo W, Soffers T, Andrinopoulou ER, et al. Quantitative assessment of airway dimensions in young children with cystic fibrosis lung disease using chest computed tomography. Pediatr Pulmonol 2017; 52: 1414-1423.

36 Kuo W, Andrinopoulou ER, Perez-Rovira A, et al. Objective airway artery dimensions compared to CT scoring methods assessing structural cystic fibrosis lung disease. J Cyst Fibros 2017; 16: 116-123.

37 Perez-Rovira A, Kuo W, Petersen J, et al. Automatic airway-artery analysis on lung CT to quantify airway wall thickening and bronchiectasis. Med Phys 2016; 43: 5736.

38 Szczesniak R, Turkovic L, Andrinopoulou ER, et al. Chest imaging in cystic fibrosis studies: what counts, and can be counted? J Cyst Fibros 2017; 16: 175-185.

39 Eichler I, Manolis E. Chest imaging in CF studies - commentary. J Cyst Fibros 2017; 16: 173-174

40 Durmowicz AG. Cystic fibrosis radiographic biomarkers - commentary. J Cyst Fibros 2017; $16: 172$.

41 Smith FW. The value of NMR imaging in pediatric practice: a preliminary report. Pediatr Radiol 1983; 13 141-147.

42 Ciet P, Tiddens HA, Wielopolski PA, et al. Magnetic resonance imaging in children: common problems and possible solutions for lung and airways imaging. Pediatr Radiol 2015; 45: 1901-1915.

43 Tepper LA, Ciet P, Caudri D, et al. Validating chest MRI to detect and monitor cystic fibrosis lung disease in a pediatric cohort. Pediatr Pulmonol 2016; 51: 34-41.

44 Roach DJ, Cremillieux Y, Fleck RJ, et al. Ultrashort echo-time magnetic resonance imaging is a sensitive method for the evaluation of early cystic fibrosis lung disease. Ann Am Thorac Soc 2016; 13: 1923-1931.

45 Dournes G, Menut F, Macey J, et al. Lung morphology assessment of cystic fibrosis using MRI with ultra-short echo time at submillimeter spatial resolution. Eur Radiol 2016; 26: 3811-3820.

46 Higano NS, Fleck RJ, Spielberg DR, et al. Quantification of neonatal lung parenchymal density via ultrashort echo time MRI with comparison to CT. J Magn Reson Imaging 2017; 46: 992-1000.

47 Stahl M, Wielputz MO, Graeber SY, et al. Comparison of lung clearance index and magnetic resonance imaging for assessment of lung disease in children with cystic fibrosis. Am J Respir Crit Care Med 2017; 195: 349-359.

48 Gulani V, Calamante F, Shellock FG, et al. Gadolinium deposition in the brain: summary of evidence and recommendations. Lancet Neurol 2017; 16: 564-570. 
49 Voskrebenzev A, Gutberlet M, Kaireit TF, et al. Low-pass imaging of dynamic acquisitions (LIDA) with a group-oriented registration (GOREG) for proton MR imaging of lung ventilation. Magn Reson Med 2017; 78: 1496-1505.

50 Flors L, Mugler JP III, de Lange EE, et al. Hyperpolarized gas magnetic resonance lung imaging in children and young adults. J Thorac Imaging 2016; 31: 285-295.

51 Walkup LL, Thomen RP, Akinyi TG, et al. Feasibility, tolerability and safety of pediatric hyperpolarized ${ }^{129} \mathrm{Xe}$ magnetic resonance imaging in healthy volunteers and children with cystic fibrosis. Pediatr Radiol 2016; 46: $1651-1662$.

52 Kanhere N, Couch MJ, Kowalik K, et al. Correlation of lung clearance index with hyperpolarized ${ }^{129}$ Xe magnetic resonance imaging in pediatric CF subjects. Am J Respir Crit Care Med 2017; 196: 1073-1075.

53 Thomen RP, Walkup LL, Roach DJ, et al. Hyperpolarized ${ }^{129} \mathrm{Xe}$ for investigation of mild cystic fibrosis lung disease in pediatric patients. J Cyst Fibros 2017; 16: 275-282.

54 Marshall H, Horsley A, Taylor CJ, et al. Detection of early subclinical lung disease in children with cystic fibrosis by lung ventilation imaging with hyperpolarised gas MRI. Thorax 2017; 72: 760-762.

55 Altes TA, Johnson M, Fidler M, et al. Use of hyperpolarized helium-3 MRI to assess response to ivacaftor treatment in patients with cystic fibrosis. J Cyst Fibros 2017; 16: 267-274.

56 Altes TA, Mugler JP III, Ruppert K, et al. Clinical correlates of lung ventilation defects in asthmatic children. J Allergy Clin Immunol 2016; 137: 789-796.

57 Donnola SB, Dasenbrook EC, Weaver D, et al. Preliminary comparison of normalized T1 and non-contrast perfusion MRI assessments of regional lung disease in cystic fibrosis patients. J Cyst Fibros 2017; 16: 283-290.

58 Flors L, Mugler JP 3rd, Paget-Brown A, et al. Hyperpolarized helium-3 diffusion-weighted magnetic resonance imaging detects abnormalities of lung structure in children with bronchopulmonary dysplasia. J Thorac Imaging 2017; 32: 323-332.

59 Hellebrandova L, Chlumsky J, Vostatek P, et al. Airflow limitation is accompanied by diaphragm dysfunction. Physiol Res 2016; 65: 469-479.

60 Sodhi KS, Khandelwal N, Saxena AK, et al. Rapid lung MRI in children with pulmonary infections: time to change our diagnostic algorithms. J Magn Reson Imaging 2016; 43: 1196-1206.

61 Dournes G, Berger P, Refait J, et al. Allergic bronchopulmonary aspergillosis in cystic fibrosis: MR imaging of airway mucus contrasts as a tool for diagnosis. Radiology 2017; 285: 261-269.

62 Ozcan HN, Gormez A, Ozsurekci Y, et al. Magnetic resonance imaging of pulmonary infection in immunocompromised children: comparison with multidetector computed tomography. Pediatr Radiol 2017; 47: 146-153.

63 Ciet $\mathrm{P}$, Bertolo S, Ros M, et al. Detection and monitoring of lung inflammation in cystic fibrosis during respiratory tract exacerbation using diffusion-weighted magnetic resonance imaging. Eur Respir J 2017; 50: 1601437

64 Leary D, Svenningsen S, Guo F, et al. Hyperpolarized ${ }^{3} \mathrm{He}$ magnetic resonance imaging ventilation defects in asthma: relationship to airway mechanics. Physiol Rep 2016; 4: e12761.

65 Sodhi KS, Bhatia A, Khandelwal N. Rapid lung magnetic resonance imaging in children with pulmonary infection. Pediatr Radiol 2017; 47: 764-765.

66 Sodhi KS, Sharma M, Saxena AK, et al. MRI in thoracic tuberculosis of children. Indian J Pediatr 2017; 84: 670-676.

67 Weis M, Zoellner FG, Hagelstein C, et al. Lung perfusion MRI after congenital diaphragmatic hernia repair in 2-year-old children with and without extracorporeal membrane oxygenation therapy. Am J Roentgenol 2016; 206: $1315-1320$.

68 Schopper MA, Walkup LL, Tkach JA, et al. Evaluation of neonatal lung volume growth by pulmonary magnetic resonance imaging in patients with congenital diaphragmatic hernia. J Pediatr 2017; 188: 96-102.

69 Gorkem SB, Kose S, Lee EY, et al. Thoracic MRI evaluation of sarcoidosis in children. Pediatr Pulmonol 2017; 52: 494-499.

70 Ciet $\mathrm{P}$, Boiselle PM, Heidinger B, et al. Cine MRI of tracheal dynamics in healthy volunteers and patients with tracheobronchomalacia. AJR Am J Roentgenol 2017; 209: 757-761.

71 Semple T, Calder A, Owens CM, et al. Current and future approaches to large airways imaging in adults and children. Clin Radiol 2017; 72: 356-374.

72 Liszewski MC, Ciet P, Sodhi KS, et al. Updates on MRI evaluation of pediatric large airways. Am J Roentgenol 2017; 208: 971-981. 